\title{
A Compact Rectangular Monopole Antenna Design with a Novel Feed for an Improved UWB Performance
}

\author{
Gopikrishna MADANAN ${ }^{1}$, Deepti Das KRISHNA ${ }^{2}$, Chandroth Karuvandy AANANDAN ${ }^{3}$ \\ ${ }^{1}$ Department of Physics, Maharaja's College, Ernakulam, Kerala, India, PIN 682301 \\ ${ }^{2}$ Department of Electronics and Communication Engineering, Rajagiri School of Engineering and Technology, Kakkanad, \\ Kerala, India, PIN 682039 \\ ${ }^{3}$ Department of Electronics, Cochin University of Science and Technology, Cochin, Kerala, India, PIN 682022
}

gopiikrishna@maharajas.ac.in, deeptidk@rajagiritech.edu.in, anand@cusat.ac.in

Submitted April 29, 2017 / Accepted October 27, 2017

\begin{abstract}
Printed wideband antennas, if not optimised both in frequency \& time domain, often fail to qualify for $U W B$ applications. This is primarily observed in designs based on multiple resonances. The excitation of different modes causes variation in the position of the antenna phase centre and radiation patterns with frequency. In this paper, we propose a novel compact UWB antenna design with optimised resonances within the 3.1-10.6 GHz band. The design comprises of a rectangular $C P W$-fed monopole. The novelty in the design lies in the impedance transformer arrangement at the antenna feed which not only maintains constant impedance over the wide band but contributes towards maintaining uniformity in the radiation patterns over the frequency band. The proposed antenna is characterized, both in the frequency and time domain, confirming its suitability for UWB operation. The impedance bandwidth of the antenna is from 2.9$11.5 \mathrm{GHz}$ with an omni-directional radiation pattern over the full band, unlike most of the planar UWB antennas reported in literature whose pattern deteriorates particularly at the upper end of the band. It also exhibits appreciable polarization purity and radiation efficiency. The time domain parameters, Full Width Half Maxima (FWHM) and Ringing, are computed from the measured antenna transfer function. The computed Fidelity of the transmitted pulses indicates superior pulse handling capabilities. The antenna is suitable for commercial hand held devices as its PCB area is just $30 \times 12 \mathrm{~mm}^{2}$ when fabricated on the FR4 substrate with permittivity 4.4 and height $1.6 \mathrm{~mm}$.
\end{abstract}

\section{Keywords}

Ultrawide-band, monopole antenna, antenna transfer function, time domain

\section{Introduction}

Ultra-wide band systems transmit and receive ultrashort electromagnetic pulses having limited effective radiated power. The systems performance is determined primarily by the characteristics of the radiators that has to conform to stringent frequency and time domain requirements in the entire operating band [1]. These requirements are namely, a nondispersive phase center; constant radiation and impedance over the frequency range with no excitation of higher order modes [2]. Most of the planar monopole antennas with a wide operating band are most often well matched multi-resonant structures. In [3], Ma et al. have described the time domain performance of a printed dipole antenna employing a tapered slot feed. It reports that the received pulses are distorted and broadened to more than $1 n s$ in spite of the antenna being well matched over the full band. This is attributed to the shift in the antenna phase centres due to the excitation of different higher order modes. However, a much lesser pulse distortion is observed in a tapered slot antenna with fewer resonances within the $3.1-10.6 \mathrm{GHz}$ band reported by the same authors in [4].

Printed antennas, that has the advantage of portability desired for commercial applications, also gives the engineer a degree of flexibility in the designs. In the present work, a rectangular monopole is chosen as the radiating element. The ground plane is optimally designed for a non dispersive antenna phase center while maintaining a wideband impedance match. The general practice to realize ultrawidebandwidth in rectangular radiating elements is to modify the ground-radiator interface. In [5], the inherent resonances of a square patch are matched over the wide band by incorporating cuts in the ground plane of the monopole radiator at specific locations. Bevelling of the radiator/ ground plane or both is another technique as illustrated in [6], [7]. A few researchers have introduced serrations in the antenna geometry to result in ultrawide-bandwidth [8-11]. In [12], the authors have proposed a novel technique to realize UWB by employing a trident shaped feed and tapered impedance transformer to the rectangular radiating element.

In this paper, we present the design of a novel CPW fed UWB rectangular monopole antenna. The designs proposed in [5] and [13] have been contemplated in designing 


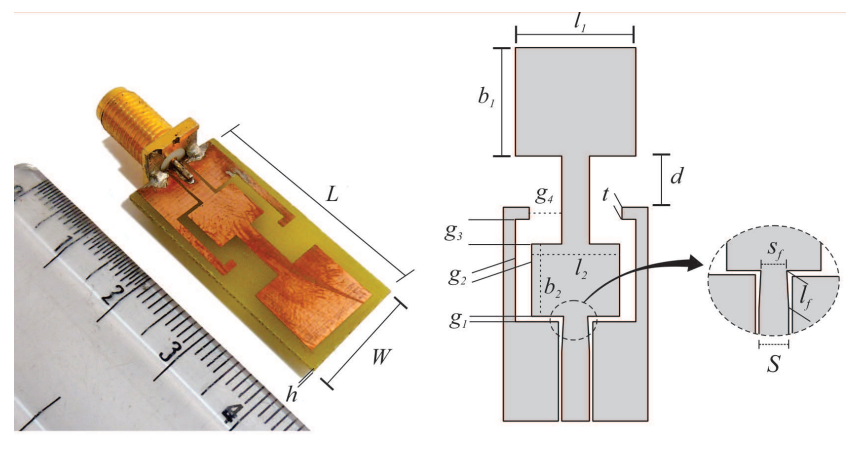

(a)

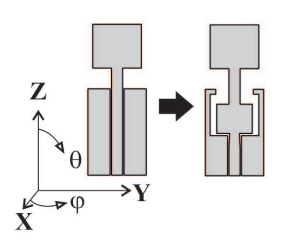

(b)

Fig. 1. (a) Configuration of the proposed antenna $s=2.3, g=$ $0.28, d=4.25, l_{1}=9, b_{1}=10, l_{g}=17.75, l_{2}=6, b_{2}=$ $7.3, g_{1}=0.45, g_{2}=1.35, g_{3}=2, g_{4}=2.7, t=1, s_{\mathrm{f}}=2$, $l_{\mathrm{f}}=3, L=30, W=12, \epsilon_{\mathrm{r}}=4.4, \tan (\delta)=0.02, h=1.6$ (all units of lenghts are in $\mathrm{mm}$ ). (b) design evolution.

the present structure. The ground plane of these kind of antennas are an active participant in the radiation process [14]. To achieve broadband matching, a wide gap is set between the rectangular radiator and the ground plane. An impedance transformer designed in the ground plane minimizes standing waves in the feed line, allowing wide impedance bandwidth from $2.9-11 \mathrm{GHz}$. Advantages of this unique design is two fold: firstly the PCB footprint is restricted to as low as $30 \times 12 \mathrm{~mm}^{2}$ which is much smaller in size when compared to the UWB antennas of this genre [11] and secondly antenna radiation patterns in the 3.1-10.6 UWB remains stable as will be shown in Sec. 3. The radiation pattern squinting in UWB antennas at higher frequencies can be attributed to the undesired current distribution on antenna ground [15]. The unique design of the ground plane of the proposed antenna inherently takes care of this detrimental effect and keeps the radiation patterns pointed along the broadside direction throughout the entire UWB.

\section{Antenna Design}

The topology of the proposed monopole antenna comprises of a rectangular monopole of area $l_{1} \times b_{1}$ as shown in Fig. 1(a). This antenna is evolved from a basic printed monopole design shown in Fig. 1(b). The coupling of the guided waves to the radiator is strongly dependent on parameter $d$ and the resonances in the structure can be matched by suitably choosing $d$.

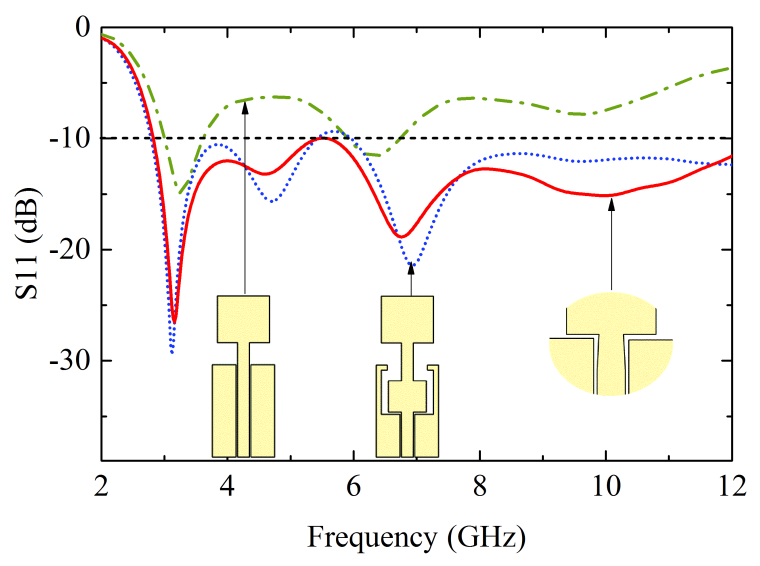

Fig. 2. Improvement in the impedance bandwidth of the antenna when the transformer is incorporated.

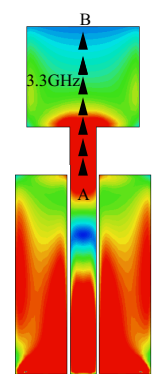

(a)

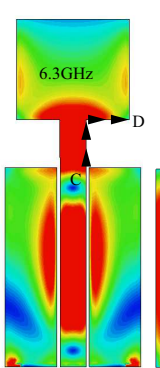

(b)

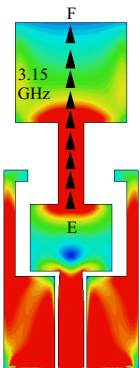

(c)

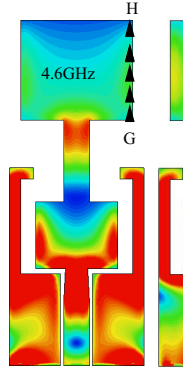

(d)

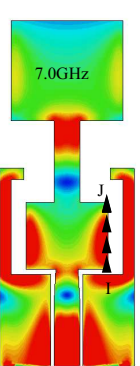

(e)
Fig. 3. Surface current plots in CST MWS.

The basic printed monopole antenna and its return loss plot is indicated in Fig. 2.To facilitate ultra wide band performance, an impedance transformer is embedded in the CPW transmission line with increasing slot widths $g_{1}, g_{2}, g_{3}$ and $g_{4}$. The special design of the transformer provide gradual transformation of the $50 \Omega$ line impedance and at the same time, result in a new current path for radiation.

Surface current plots of the simple CPW fed monopole shown in Fig. 3(a) and (b) indicates that a quarter wave variation of the current along paths $A B$ and $C D$ result in the corresponding resonances at 3.3 and $6.3 \mathrm{GHz}$. When the transformer is incorporated in the design, the first current path remains nearly the same. The second current path $G H$ is due to the rectangular monopole itself, which was not present in the simple CPW fed monopole due to the finite distance $d$ between its ground and the radiator. The third resonance in the structure is due to a variation of the current path $I J$. All these resonances are impedance matched to result in ultrawide band and a slight mismatch at the lower frequencies is taken care by designing a taper at the end of the feed with parameters $s_{\mathrm{f}}$ and $l_{\mathrm{f}}$. It is observed that the proposed design has an impedance bandwidth that covers the $3.1-10.6 \mathrm{GHz}$ UWB as shown in Fig. 2. For the studies presented in this paper, FR4 glass epoxy with relative permittivity $\epsilon_{\mathrm{r}}=4.4$, dielectric loss tangent $\tan (\delta)=0.02$ and laminate thickness $h=1.6 \mathrm{~mm}$ is used as the substrate. 


\section{Frequency Domain Characteriza- tions}

Frequency domain measurements of the prototype antenna was performed using R\&S ZVB 20 Vector Network Analyzer. The measured return loss indicates broad impedance bandwidth from $2.9-11 \mathrm{GHz}$ as shown in Fig. 4. The simulated resonances are in good agreement with the measurement.

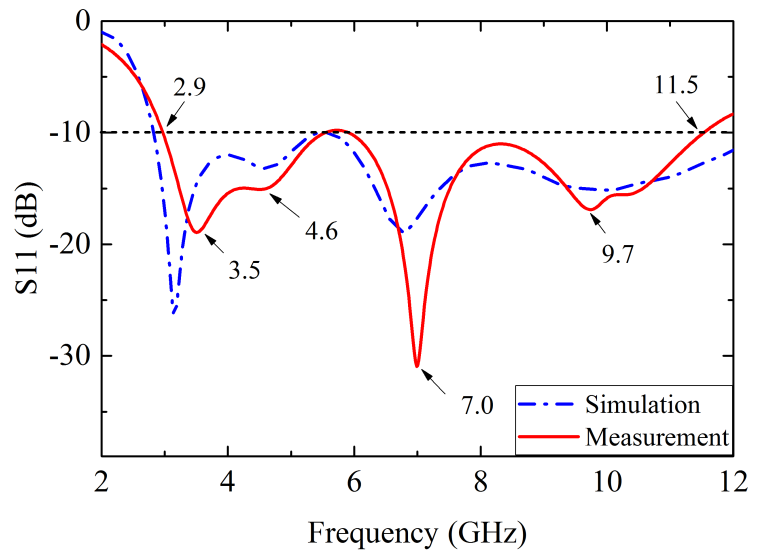

Fig. 4. Measured and simulated return loss.

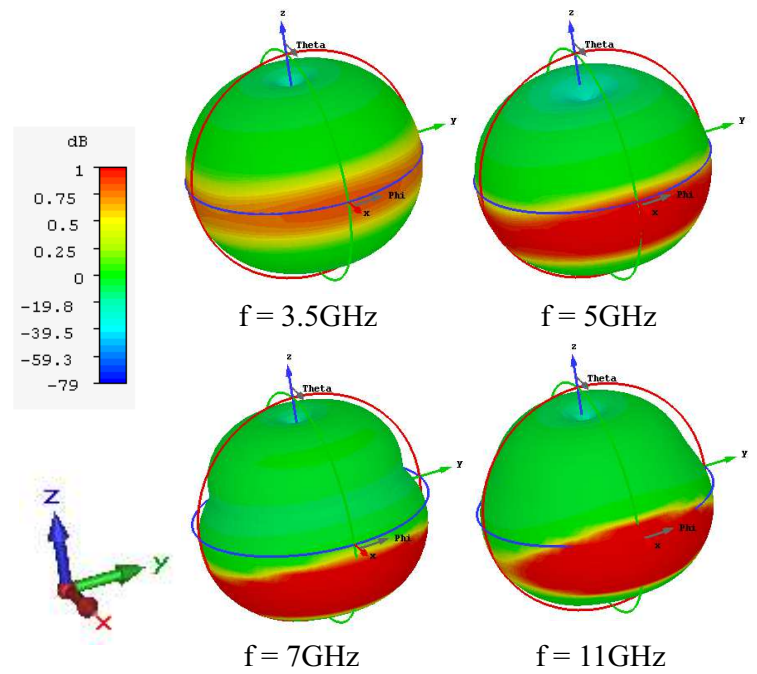

Fig. 5. 3-D radiation patterns of the proposed antenna.

Stable radiation patterns are highly desired for UWB antennas. 3-D radiation patterns obtained from CST (Fig. 5) indicates that pattern degradation at the higher frequencies are minimum; radiation is stable and directed towards the boresight at all frequencies. Measured radiation patterns at 3.5, 7 and $11 \mathrm{GHz}$ are shown in Fig. 6. Azimuthal patterns of the antenna remains omni-directional throughout the available bandwidth and pattern degradation at higher frequencies is minimal (Fig. 6(a)). The corresponding elevation patterns remain quasi omni-directional (Figure 6(b)). Cross-polar level exhibited by the antenna remains better than $-20 \mathrm{~dB}$ in the $3.1-10.6 \mathrm{GHz}$ band. Antenna polarization is linear and is oriented in the $\mathrm{Z}$ direction. Peak gains of the antenna measured by gain transfer method is compared with simulations

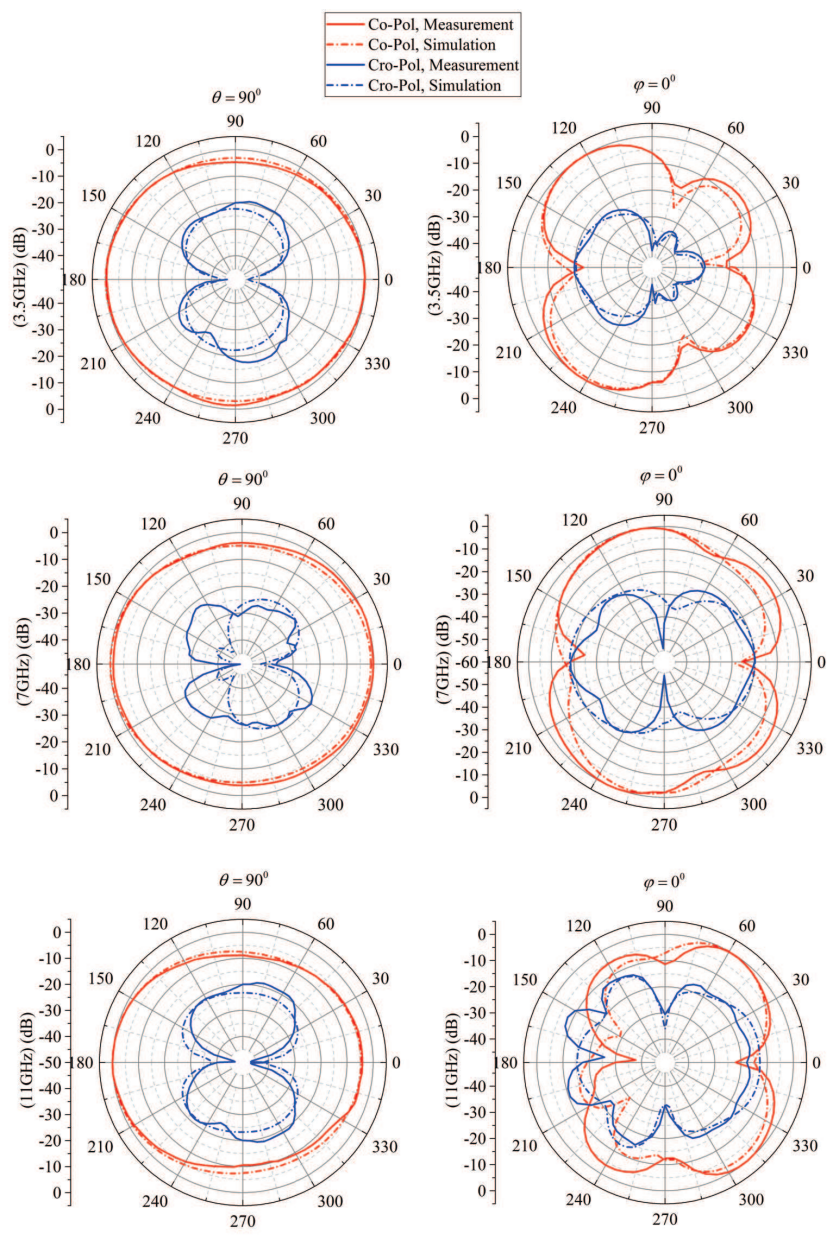

(a)

(b)

Fig. 6. Measured and simulated radiation patterns in the (a) azimuthal (X-Y) (b) elevation (X-Z) plane at 3.5, 7.0, and $11.0 \mathrm{GHz}$.

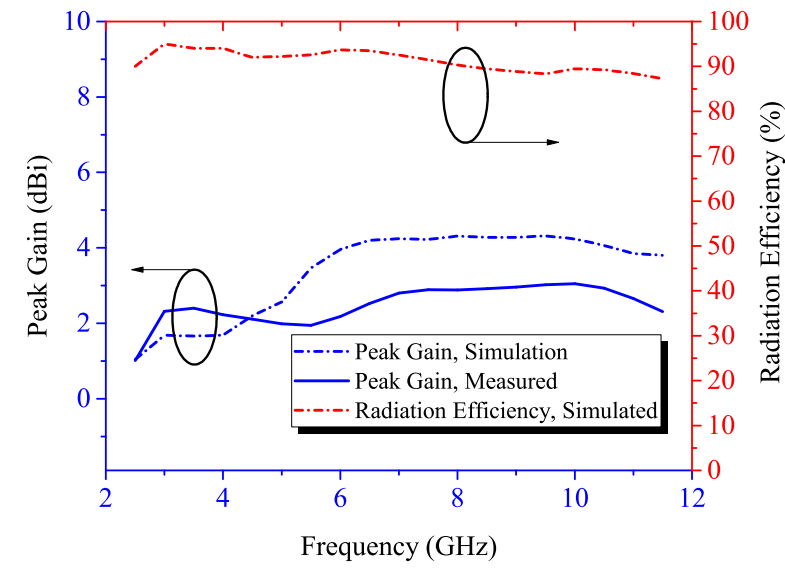

Fig. 7. Measured and simulated peak gain and radiation efficiency.

and is shown in Fig. 7. Peak gains are in the broad side direction and its average value in the $3.1-10.6 \mathrm{GHz}$ UWB is $2.6 \mathrm{dBi}$. Radiation efficiency of the antenna obtained from simulations are found to be appreciable and its average value in the UWB is $91 \%$. 


\section{Time Domain Characterizations}

Temporal characteristics of an antenna is a key parameter in determining its suitability for use in UWB systems. High fidelity reception or transmission of a wide-band signal is often impaired by antenna geometry as antennas that are well matched in the frequency domain may behave differently in the time domain.

To characterize the rectangular strip monopole antenna in the time domain, it is modelled as a linear time invariant system defined by a transfer function and associated impulse response. If the antenna phase center is dispersive, the effective group delay will be distorted in the frequency domain. The amplitude and phase of the antenna transfer function in the transmitting mode is a function of the antenna gain and group delay. Any deviation in the antenna behaviour in the frequency domain will be reflected in the antenna transfer plots and in its pulse preserving capabilities [18].

\subsection{Computation}

In [17], Duroc et al. have proposed a model of UWB channel propagation considering the transmit antenna $\left(H_{\mathrm{Tx}}(\omega, \theta, \varphi)\right)$, receive antenna $\left(H_{\mathrm{Rx}}(\omega, \theta, \varphi)\right)$ and free space channel $\left(H_{\mathrm{Ch}}(\omega)\right)$ transfer functions. These are related to the coupling parameter $S_{21}(\omega, \theta, \varphi)$ in the frequency domain as

$$
\begin{gathered}
H_{\mathrm{Tx}}(\omega, \theta, \varphi)=\sqrt{\frac{\mathrm{j}}{2 \pi}\left(\frac{\omega}{c}\right)^{2} \frac{S_{21}(\omega, \theta, \varphi)}{H_{\mathrm{Ch}}(\omega, \theta, \varphi)}}, \quad\left[\mathrm{m}^{-1}\right] \\
H_{\mathrm{Rx}}(\omega, \theta, \varphi)=\sqrt{\frac{2 \pi}{\mathrm{j}}\left(\frac{c}{\omega}\right)^{2} \frac{S_{21}(\omega, \theta, \varphi)}{H_{\mathrm{Ch}}(\omega, \theta, \varphi)}}, \quad[\mathrm{m}] \\
H_{\mathrm{Ch}}(\omega)=\frac{c}{2 d^{\prime} \omega} \exp \left(\frac{-\mathrm{j} \omega d^{\prime}}{c}\right)
\end{gathered}
$$

where $c$ is the velocity of the light, $\omega=2 \pi f$ is the angular frequency and $d^{\prime}$ is the distance between the antennas.

Three antennas are required for measurements; two identical wide band ridged horn antennas and the test antenna. The transmit antenna used for the measurements is first standardized using its identical pair. After this, the wide band ridged horn at the receiving end is replaced with the test antenna and the transmission coefficient is measured for its multiple orientations. The transfer function of the test antenna is computed using,

$$
H_{\mathrm{AUT}}(\omega, \theta, \varphi)=\frac{S_{21}(\omega, \theta, \varphi)}{H_{\mathrm{Tx}}(\omega) H_{\mathrm{Ch}}(\omega)} .
$$

The detailed account of the data processing done in MATLAB explained in [19]. From the antenna transfer function, impulse response is computed by performing an IFFT.

\subsection{Time Domain Parameters}

Antenna effects on the transmitted / received signals are investigated in the time domain by analyzing the envelope of the analytic response for co-polarization. To measure the linear distortion of the antenna, magnitude of the transient response envelope known as full width at half maximum (FWHM) is computed. Another parameter ringing is defined as the time until the envelope has fallen from the peak value below certain fraction of the main peak. The analytic response can be convoluted with an assumed input pulse to obtain effects of the antenna on the received signals as a waveform distortion. The distortion in the pulse is accounted by computing the fidelity as [16]

$$
F=\max _{\tau}\left\{\frac{\int_{-\infty}^{+\infty} s_{i}(t) \cdot s_{\mathrm{o}}(t-\tau) \mathrm{d} t}{\sqrt{\int_{-\infty}^{+\infty}\left|s_{i}(t)^{2}\right| \mathrm{d} t \cdot \int_{-\infty}^{+\infty}\left|s_{\mathrm{O}}(t)^{2}\right| \mathrm{d} t}}\right\} .
$$

For the present study, the input pulse taken is the fourth derivative of the Gaussian pulse with mathematical form [19]

$$
\begin{array}{r}
s_{i}(t)=A \cdot\left[3-6\left(\frac{4 \pi}{T^{2}}\right)(t-\tau)^{2}+\left(\frac{4 \pi}{T^{2}}\right)^{2}(t-\tau)^{4}\right] \\
\times \mathrm{e}^{-2 \pi\left(\frac{t-\tau}{T}\right)^{2}} \cdot\left[\frac{\mathrm{V}}{\mathrm{m}}\right]
\end{array}
$$

This pulse conforms to the FCC spectral mask when $A=0.333$ and $T=0.175 \mathrm{nS}$.

\subsection{Computed Time Domain Results}

As an UWB signal is transmitted or received, the pulse shape is altered. This distortion is reflected in the transfer function (in the frequency domain) or impulse response (in the time domain) of the antenna. The antenna transfer function has an amplitude as well as phase and hence is a complex quantity. In the case when the antenna has no effect on the pulse transmitted, the transfer function would be constant in phase and amplitude with frequency while the impulse response would be a delta function. For the proposed design, the measured transfer function in the azimuthal plane is almost constant at all spatial angles and the variation remains within $10 \mathrm{~dB}$ (Fig. 8(a)). This indicates that the antenna induced transmitted/ received signal variations are minimum and this is further confirmed by the corresponding impulse response indicated in Fig. 8(c) that resemble a delta function. The ripples seen post the maximum peak in the same figure is the ringing effect which can be seen to settle fast. The measured transfer functions in the elevation plane (Fig. 8(b)) shows nulls at $0,60,180$ and 300 degrees. This result is in agreement with the radiation patterns shown in Fig. 6(b).

The corresponding impulse responses found to contain variations in the peak amplitudes as well as pronounced ripples (Fig. 8(d)).

In order to understand the versatility of the antenna in pulse handling, its performance is compared with that of the Square monopole antenna described in [5]. The frequency domain performance of the antennas are similar as explained in Tab. 1, but they differ in PCB dimension. Fig. 9 and 10 shows the time domain performace indicators of the antennas for azimuthal and elevation planes. For the rectangular 


\begin{tabular}{|l|l|l|l|l|l|l|}
\hline Antenna & $\begin{array}{l}\text { Bandwidth } \\
{[\mathrm{GHz}] /[\% \mathrm{BW}]}\end{array}$ & $\begin{array}{l}\text { Resonances } \\
{[\mathrm{GHz}]}\end{array}$ & Pattern & $\begin{array}{l}\text { Average gain [dBi]/ } \\
\text { Radiation efficiency [\%] }\end{array}$ & Laminate & $\begin{array}{l}\text { Size } \\
{\left[\mathrm{mm}^{2}\right]}\end{array}$ \\
\hline $\begin{array}{l}\text { Square monopole an- } \\
\text { tenna [5] }\end{array}$ & $2.9-15 / 145$ & $3.75,8$ & $\begin{array}{l}\text { Omni direc- } \\
\text { tional }\end{array}$ & $3.75 / 86$ & $\begin{array}{l}\text { FR4 Glass Epoxy } \\
\epsilon_{\mathrm{r}}=4.4, h=1.6 \mathrm{~mm}\end{array}$ & $29.5 \times 32$ \\
\hline Present work & $2.9-11.5 / 119$ & $3.5,7$ & $\begin{array}{l}\text { Omni direc- } \\
\text { tional }\end{array}$ & $2.6 / 91$ & $\begin{array}{l}\text { FR4 Glass Epoxy } \\
\epsilon_{\mathrm{r}}=4.4, h=1.6 \mathrm{~mm}\end{array}$ & $30 \times 12$ \\
\hline
\end{tabular}

Tab. 1. Comparison of the rectangular monopole anntenna with square monopole antenna in [5].

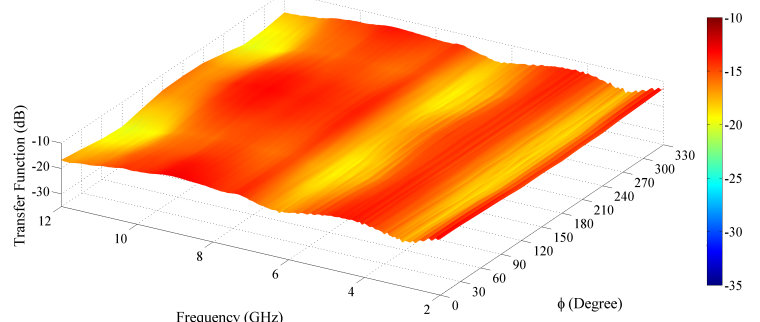

(a)

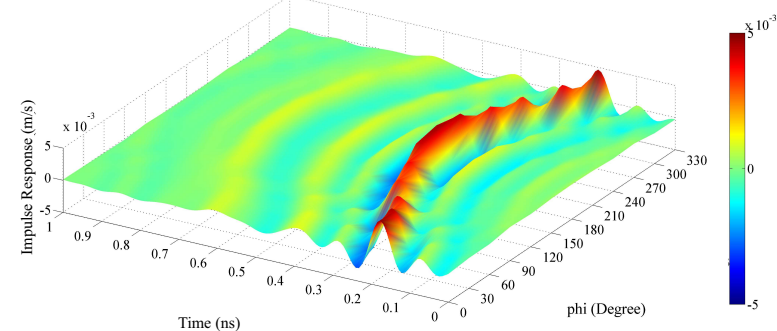

(c)

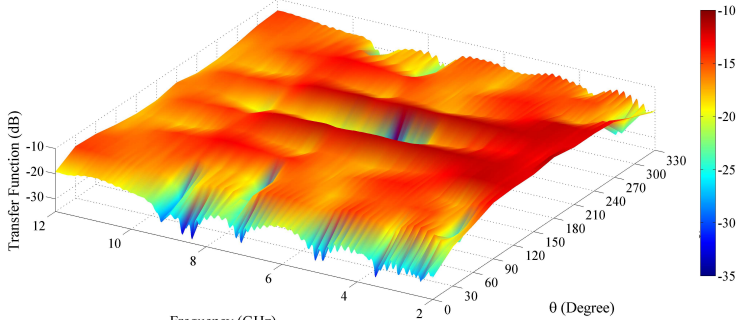

(b)

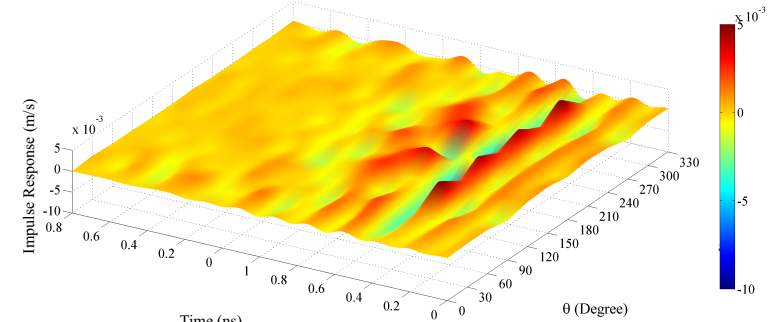

(d)

Fig. 8. Antenna transfer function measured in the (a) azimuthal (X-Y) (b) elevation (X-Z); Antenna impulse response measured in the (a) azimuthal $(\mathrm{X}-\mathrm{Y})(\mathrm{b})$ elevation $(\mathrm{X}-\mathrm{Z})$ planes for multiple orientations of the test antenna.

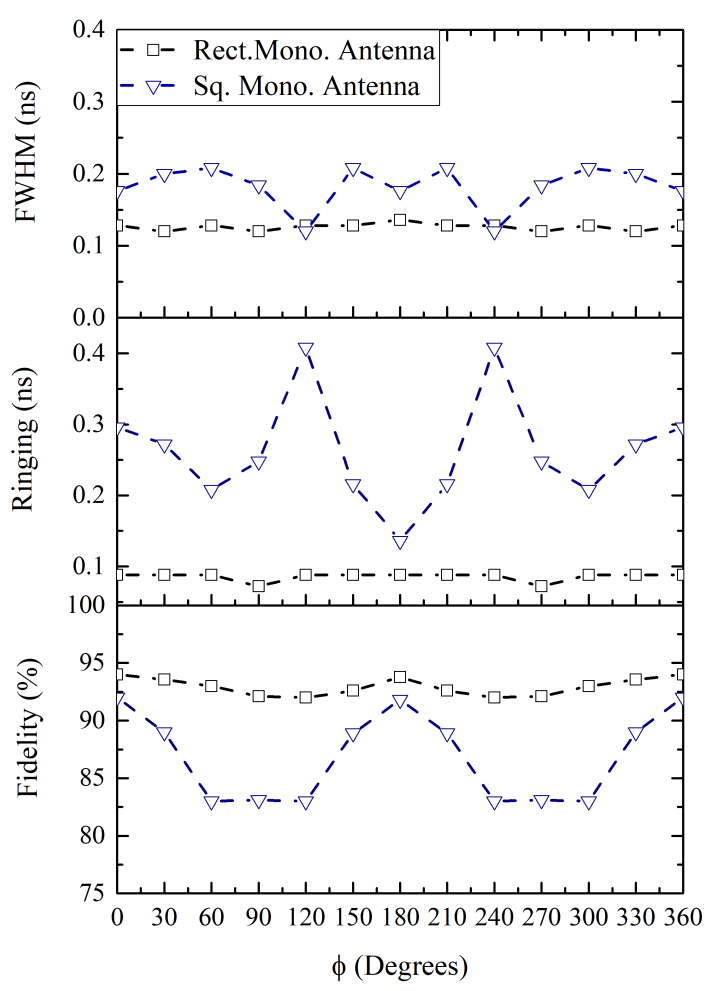

Fig. 9. Computed (a) FWHM (b) ringing and (c) fidelity in the azimuthal plane.

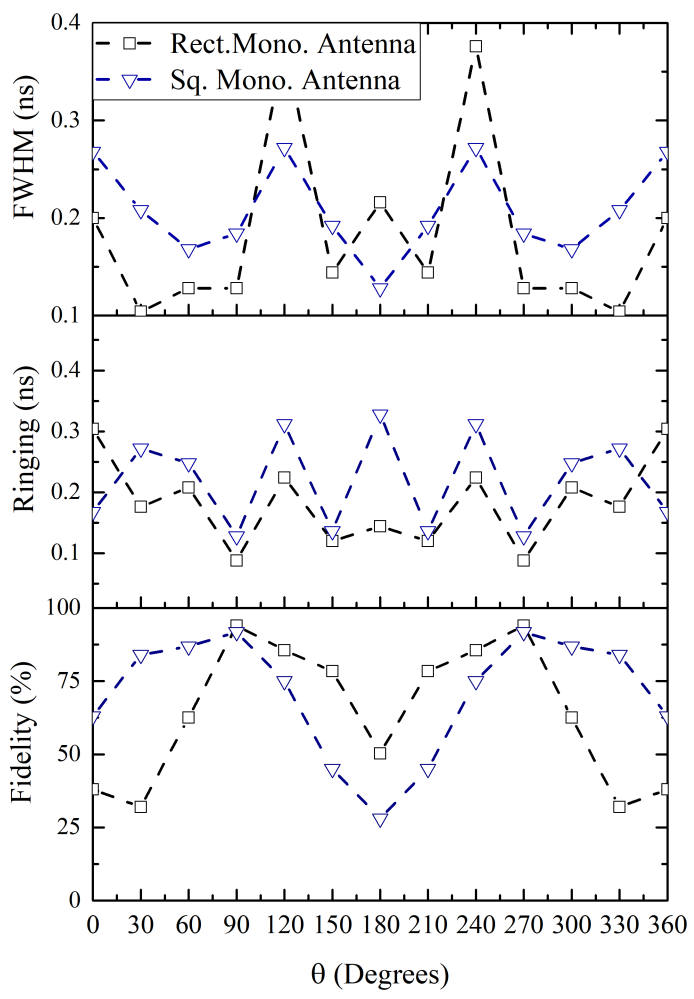

Fig. 10. Computed (a) FWHM (b) ringing and (c) fidelity in the elevation plane. 
monopole antenna, the FWHM is constant and ringing is minimum at all angles in the azimuthal plane as observed from Fig. 9. Though within acceptable limits, the square monopole antenna however shows variations which could be attributed to its relatively larger size. Computations also indicate that fidelity of the received pulses of the rectangular monopole is constant and better compared to the square monopole.

Hence, from the present study, it can be concluded that for good time domain performance, resonances in the antenna within the operating band as well as physical size of the antenna has to be minimum; the former condition will minimize internal reflections in the antenna geometry while the latter will make sure that there are no changes in the phase centre of the antenna. The performance indicators show significant variations in the elevation plane (Fig. 10), but that is expected due to the nulls in the radiation at $\left(\theta=0^{\circ}, \phi=0^{\circ}\right)$ and $\left(\theta=180^{\circ}, \phi=0^{\circ}\right)$ directions.

\section{Conclusion}

Printed UWB antennas are multi-resonant structures that operate over wide frequency range. However, these elements may often result in travelling phase centers impairing their use in time domain applications. The variation in antenna phase center can be quantified in terms of group delay or antenna transfer function, a curb on the former being $1 \mathrm{nS}$ over the entire band and that on the latter is to remain within $10 \mathrm{~dB}$. Through the novel rectangular monopole antenna presented in this paper, we have demonstrated that printed antennas with minimum resonances and smaller physical size will have superior time domain performance compared to their multi-resonant, sizeable contenders. Advantages of the optimally designed ground plane of the present antenna is manifold: (i) wide impedance bandwidth from $2.9-11.5 \mathrm{GHz}$ (ii) compact physical size of $30 \times 12 \mathrm{~mm}^{2}$ (iii) minimum phase center variation that result in superior radiation even in the higher UWB and (iv) superior transient performance indicators. These characteristics makes the novel rectangular monopole antenna proposed in this paper a good candidate for multimode and UWB applications.

\section{Acknowledgments}

Simulation and measurements of the present work were performed at the facilities created with the financial aid of UGC, DST-FIST and KSCSTE at the Centre for Electromagnetic Research and Antennas (CREMA), Dept. of Electronics, Cochin University of Science and Technology, Cochin, Kerala, India.

\section{References}

[1] GHOSH, D., DE, A., TAYLOR, M. C., et al. Transmission and reception by ultra-wideband (UWB) antennas. IEEE Antennas and Propagation Magazine, 2006, vol. 48, no. 5, p. 67-99. DOI: 10.1109/MAP.2006.277157
[2] GALVAN-TEJADA, G. M., PEYROT-SOLIS, M. A., AUGILAR, H. J. Ultra Wideband Antennas: Design, Methodologies, and Performance. CRC Press, 2015. ISBN: 9781482206500

[3] MA, T. G., JENG, S. K. A printed dipole antenna with tapered slot feed for ultrawide-band applications. IEEE Transactions on Antennas and Propagation, 2005, vol. 53, no. 11, p. 3833-3836. DOI: 10.1109/TAP.2005.858819

[4] MA, T. G., JENG, S. K. Planar miniature tapered-slot-fed annular slot antennas for ultrawide-band radios. IEEE Transactions on Antennas and Propagation, 2005, vol. 53, no. 3, p. 1194-1202. DOI: $10.1109 /$ TAP.2004.842648

[5] GOPIKRISHNA, M., KRISHNA, D. D., CHANDRAN, A. R., et al. Square monopole antenna for ultra wide band communication applications. Journal of Electromagnetic Waves and Applications, 2007, vol. 21 , no. 11 , p. $1525-1537$. DOI: $10.1163 / 156939307782000299$

[6] CHU, Q. X., YANG, Y. Y. A compact ultrawideband antenna with 3.4/5.5 GHz dual band-notched characteristics. IEEE Transactions on Antennas and Propagation, 2008, vol. 56, no. 12, p. 3637-3644. DOI: 10.1109/TAP.2008.2007368

[7] ACHARJEE, J., MANDAL, K., MANDAL, S. K., et al. A compact printed monopole antenna with enhanced bandwidth and variable dual band notch for UWB applications. Journal of Electromagnetic Waves and Applications, 2016, vol. 30, no. 15, p. 1980-1992. DOI: $10.1080 / 09205071.2016 .1234419$

[8] LEE, S. S., CHOI, S. S., PARK, J. K., et al. Experimental study of UWB antenna in the time domain. Microwave and Optical Technology Letters, 2005, vol. 47, no. 6, p. 554-558. DOI: 10.1002/mop.21228

[9] SHAMEENA, V. A., SUMA, M. N., RAJ ROHITH, K., et al. Compact ultra-wideband planar serrated antenna with notch band ON/OFF control. Electronics Letters, 2006, vol. 42, no. 23, p. 1323-1324. DOI: $10.1049 / \mathrm{el}: 20062863$

[10] WU, Q., JIN, R., GENG, J., et al. Ultra-wideband rectangular disk monopole antenna with notched ground. Electronics Letters, 2007, vol. 43, no. 11, p. 605-6. DOI: 10.1049/el:20070910

[11] LI, Y., LI, W.,YE, Q. A reconfigurable triple notch band antenna integrated with defected microstrip structure bandstop filter for ultrawideband cognitive radio applications. International Journal of Antennas and Propagation, 2013, vol. 2013, p. 1-13. DOI: $10.1155 / 2013 / 472645$

[12] WU, Q., JIN, R., GENG, J., DING, M. Printed omni-directional UWB monopole antenna with very compact size. IEEE Transactions on Antennas and Propagation, 2008, vol. 56, no. 3, p. 896-899. DOI: 10.1109/TAP.2008.917018

[13] GOPIKRISHNA, M., KRISHNA, D. D., ANANDAN, C. K., et al. Design of a compact semi-elliptic monopole slot antenna for UWB systems. IEEE Transactions on Antennas and Propagation, 2009, vol. 57, no. 6, p. 1834-1837. DOI: 10.1109/TAP.2009.2015850

[14] ANGUERA, J., ANDUJAR, A. Ground plane contribution in wireless handheld devices using radar cross section analysis. Progress in Electromagnetics Research, 2012, vol. 26, p. 101-114. DOI: 10.2528/PIERM12081704

[15] FEREIDOONY, F., CHAMAANI, S., MIRTAHERI, S. A. UWB monopole antenna with stable radiation pattern and low transient distortion. IEEE Antennas and Wireless Propagation Letters, 2011, vol. 10, p. 302-305. DOI: 10.1109/LAWP.2011.2141106

[16] SORGEL, W., WIESBECK, W. Influence of the antennas on the ultrawideband transmission. Eurasip Journal on Applied Signal Processing, 2005, p. 296-305. DOI: 10.1155/ASP.2005.296

[17] DUROC, Y., GHIOTTO, A., VUONG, T. P., et al. UWB antennas: Systems with transfer function and impulse response. IEEE Transactions on Antennas and Propagation, 2007, vol. 55, no. 5, p. 14491451. DOI: $10.1109 /$ TAP.2007.895636 
[18] KWON, D. H. Effect of antenna gain and group delay variations on pulse-preserving capabilities of ultrawideband antennas. IEEE Transactions on Antennas and Propagation, 2006, vol. 54, no. 8 , p. 2208-2215. DOI: 10.1109/TAP.2006.879189

[19] GOPIKRISHNA, M., AANANDAN, C. K. On The Radiation Characteristics of Planar Printed UWB Antennas. Scholars Press, 2013. ISBN: 978-36-395-1104-8

\section{About the Authors ...}

Gopikrishna MADANAN (corresponding author) was born in India. He received his MSc. degree from the Department of Physics, Cochin University of Science and Technology (CUSAT), Cochin, India in the year 2002 and Doctoral degree in Microwave Electronics from the Department of Electronics, CUSAT in the year 2011. Presently, he is with the Department of Collegiate Education, Govt. of Kerala, India as Assistant Professor of Physics, being posted to work at the Maharaja's College, Ernakulam, India . His research interests include UWB antennas, Metamaterial resonators and Planar filters.

Deepti Das KRISHNA was born in India. She received her M.Sc. degree in Electronics and M.Tech. degree in
Microwave Electronics from Delhi University, New Delhi, India, during 2001 and 2002 respectively and Doctoral degree in in Microwave Electronics from the Department of Electronics, from Cochin University of Science and Technology (CUSAT) in the year 2011. Presently, she is Professor of Electronics and Communication Engineering at the Rajagiri School of Engineering and Technology (RSET), Cochin, India. Her research areas include UWB Antennas, Reconfigurable Antennas, Microwave Passive Devices and Communication Systems.

Chandroth Karuvandy AANANDAN was born in India. He received the M.Sc and Ph.D degrees from Cochin University of Science and Technology (CUSAT) in 1981 and 1987 respectively. Currently, he is Professor at the Department of Electronics, CUSAT. He has published many research papers in diverse area pertaining to microwave electronics in peer-reviewed journals. Prof. Aanandan is a member of IEEE, European Microwave Association, IEEE Antennas \& Propagation Society, Indian Society of Technical Education, Plasma Science Society of India and Chairman of IEEE AP-S Kerala Chapter. His research interests include microstrip antennas, UWB antennas, fractal antennas, radar cross section studies and frequency selective surfaces. 